\title{
ANÁLISIS DE LA POLÍTICA DE LAS UNIVERSIDADES ECUATORIANAS INTERVENIDAS
}

AUTORES: Freddy Aníbal Marcillo Merino ${ }^{1}$

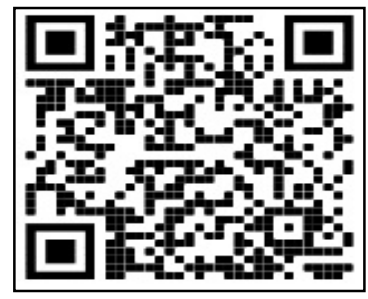

\section{DIRECCIÓN PARA CORRESPONDENCIA: fmarcillo2010@yahoo.es}

Fecha de recepción: 23/10/2020

Fecha de aceptación: 25/11/2020

\section{RESUMEN}

Un enfoque al análisis de las políticas, está presente en las universidades Ecuatorianas, basta con saber y conocer que en la actualidad hay cinco (5) instituciones de formación académica superior intervenidas, bajo un régimen diferente a los nuestros, es decir totalmente autoritario y bajo esta consigna no se puede liderar. Si bien es cierto que son pocas las instituciones de educación superior, que por primera vez se dan estos casos en el Ecuador. Por esta razón se siente la necesidad de realizar este trabajo; ante tantos paradigmas se ha analizado bibliografía y según lo investigado, utilizando el método de la encuesta se ha llegado a la conclusión de que no hay líderes con conocimientos, honestidad, moral y ética que muchos la pregonan pero no la practican rectitud que ejerzan un liderazgo eficaz que ayuden a llegar a metas planeadas y no marcar metas, por este motivo los que han venido existiendo han sido jefes, personas que solo han llevado a la destrucción es decir a un estrepitoso fracaso a la entidad que dirigen provocando incertidumbre y dejando de entre dicho la rectitud del lugar intervenido. El proceso del trabajo se realiza mediante investigación visual descriptiva, la misma que es interpretada de manera estadística en gráficos y tablas; dadas a conocer mediante la redacción concientizada de los resultados encontrados, los mismos que han dado como respuesta que del total de las personas encuestadas que conocen la diferencia entre líderes y jefes, están de acuerdo que en las universidades no hay liderazgo por ser tan evidente y palpable los actos y las intervenciones, encontrando actos relacionados con corrupción debido al mal manejo de las asignaciones del gobierno, nepotismo y varias acciones que se van a detallar en el transcurso de este trabajo. Al conocer que los lideres no nacen se hacen deberíamos utilizar eso para implementar un sistema de dirección enfocado a valores que es ahí donde al parecer se falla, uno hace lo que aprende y absorbe lo que el entorno le da, esperemos darle algo mejor a nuestras futuras generaciones para que todo cambie para bien.

PALABRAS CLAVE: Falta de Líderes; Corrupción; Honestidad; Ética; Moral; Estrepitoso Fracaso.

\section{ANALYSIS OF THE POLICY OF THE INTERVENED ECUADORIAN UNIVERSITIES}

\footnotetext{
${ }^{1}$ Universidad Estatal Del Sur De Manabí, Jipijapa, Ecuador. E-mail: fmarcillo2010@yahoo.es
} 


\begin{abstract}
One approach to policy analysis is present in Ecuadorian universities, just know and understand that there are currently five (5) institutions of higher academic training intervened under a different regime than ours that is totally authoritarian and under this slogan can not lead. While it is true that few institutions of higher education, these cases occur for the first time in Ecuador, it is why you feel the need to do this work; with so many paradigms are analyzed little bibliography and as investigated using the method of the survey has been concluded that there are no leaders with knowledge, honesty, moral and ethical many the touting but do not practice righteousness exercise effective leadership to help reach planned goals and not scoring goals, which is why those who have been there have been leaders, people who have only led to the destruction that is a dismal failure the entity directing causing uncertainty and leaving from that place straightness intervened. The process of work is done by descriptive visual research, the same that is interpreted statistically in graphs and tables; released by concentric writing of the results found the same that have been in response to the total of respondents who know the difference between leaders and managers, they agree that in universities there is no leadership for being so obvious and palpable actions and interventions, finding related to corruption due to mismanagement of government allocations, nepotism and several actions that will be detailed in the course of this work acts. . Knowing that leaders are not born are made should use that to implement a management system focused on values that is where it fails to appear, you do what you learn and absorb what the environment gives hopefully give something better to our future generations so that everything will change for the better.
\end{abstract}

KEYWORDS: Falta de Líderes, Corrupción, Honestidad, Ética, Moral, Estrepitoso Fracaso.

\title{
INTRODUCCIÓN
}

Potenciar el Liderazgo es una necesidad urgente ante la evidente crisis social que envuelve a las Universidades Ecuatorianas es un reto que se debe asumir con sentido de responsabilidad quienes de una u otra manera están sumido. El liderazgo es algo que muchos sabemos no está presente en las universidades, ¿Por qué?, basta con saber y conocer que en la actualidad hay 5 instituciones de formación académica superior intervenidas, bajo un régimen diferente a los nuestros es decir totalmente autoritario y bajo esta consigna no se puede liderar por esta razón se sintió la necesidad de realizar este trabajo; ante tantos paradigma se ha analizado mucha bibliografía y según lo investigado se ha establecido que no hay líderes que ejerzan un liderazgo eficaz que ayude llegar a metas planeadas y no marcar metas en donde se ha llegado, por este motivo los que han venido existiendo han sido jefes, personas que solo han llevado a la destrucción es decir a un estrepitoso fracaso a la entidad que dirigen provocando incertidumbre y dejando de entre dicho la rectitud que es lo que se necesita.

Al darnos cuenta que el fracaso de las universidades, es la falta de líderes acentuamos la propuesta de que para que haya lideres debe haber conocimiento del liderazgo, el mismo que debe ir de la mano con la honestidad, la rectitud, de moral de principios y sobre todo de ética que muchos la pregonan pero no la practican, de igual manera debe ir de la mano con el conocimiento de la administración. El proceso de este trabajo se lo realiza mediante investigación visual descriptiva, a los involucrados, la misma que es interpretada de manera estadística en gráficos y tablas; dadas a conocer mediante la redacción concientizada de los resultados encontrados los 
mismos que han dado como respuesta que del total de las personas encuestadas que conocen la diferencia entre líderes y jefes, consideran o están de acuerdo que en las universidades no hay liderazgo por ser tan evidente y palpable debido a las causas del por qué, varias universidades han sido intervenidas.

En estas universidades intervenidas lo que más se ha encontrado han sido actos relacionados con corrupción debido al mal manejo de las asignaciones presupuestarias del gobierno, nepotismo y varias acciones que son de conocimiento público. Al conocer que los líderes no nacen se hacen deberíamos utilizar eso para implementar un sistema de dirección enfocado a valores que es ahí donde al parecer se falla, uno hace lo que aprende y absorbe lo que el entorno le da esperemos darle algo mejor a nuestras futuras generaciones para que todo cambie para bien.

Desde esta perspectiva de sustentabilidad, el relieve y comportamiento de la naturaleza, permite realizar una propuesta para entender y solucionar el siguiente problema: Insuficiencia de líderes en la política de las universidades ecuatorianas intervenidas, de igual manera el presente artículo determina el siguiente objeto: La falta de líderes en la política de las Universidades Ecuatorianas intervenidas campo: El proceso de formación de líderes a través de la asignatura Liderazgo Organizacional, como materia integradora. Hipótesis: Si se instrumenta una estrategia didáctica asistida por un modelo de formación de una capacitación sobre Liderazgo Organizacional, en el cual exista un proceso sinérgico entre subsistema conceptuales y metodológicos, mediados por el proceso de contextualización de liderazgo objetivo: Instrumentar una estrategia didáctica de capacitación y formación de Liderazgo Organizacional para Autoridades del nivel superior desde la asignatura de Liderazgo Organizacional.

La presente investigación por naturaleza se enmarca en un diseño transversal descriptivo. Es transversal por que aborda el inconveniente precisamente en el momento que ocurre la problemática, es descriptiva ya que identifica cada uno de los rasgos que caracteriza el problema establecido en el contexto de estudio.

El nuevo contexto tecnológico obliga a la administración de la educación a reinterpretarse como profesión, de manera que le corresponde asumir en forma estratégica e indispensable los retos en los que la educación se ve insertada. Dentro de la contingencia cultural y la era digital debe considerar la gestión moderna de la educación en la conducción de las organizaciones educativas, los impactos político, económico, cultural y social que intervienen para que el proceso educativo aspire a ser de calidad y equidad, en el cual la educación inclusiva y la interculturalidad se manifiesten dentro de sus prioridades.

Haciendo alusión a lo anteriormente expuesto, es pertinente señalar que el docente debe actuar en todo momento apegado a las normas éticas de su profesión, ya que este es modelo a seguir por sus alumnos. En tal sentido, (Marques et al., 2005) expresa que "La ética profesional del maestro tiene una gran tarea en la sociedad, ya que es la responsable de la formación de las nuevas generaciones". Del mismo modo, considera que la ética pedagógica estudia el carácter de la actividad moral del pedagogo y las relaciones morales en el medio pedagógico. Por otra parte ésta ética refleja la necesidad de una sólida preparación del docente para impartir clase con calidad. De aquí que el desempeño profesional independientemente del área que se ejerza requiere ser guiado por los principios y valores éticos fundamentales en las profesiones. 
Por otro lado, (Angulo \& Acuña, 2005) "La ética es el ideal de la conducta humana, orientando sobre lo que es bueno y correcto, ésta se consolida cuando se internalizan las normas sin que exista presión exógena para su cumplimiento. La ética de un profesional, se gesta desde su formación, por ello el docente debe actuar en esta etapa de forma correcta, y para realizar esta labor tiene que conocer de ética y de cómo debe ser su comportamiento como docente" (p.1).

El profesional y más si es de la educación, debe desarrollar su trabajo basado en valores y principios éticos, ya que como guía moldea el comportamiento a través de su actuar, siendo modelo a seguir y en este sentido requiere de una sólida formación, de un perfil adecuado y de unos principios básicos que les permitan vencer cualquier problema que se le presente. (Cornelio \& Gulín, 2018), (Guzmán, 2004) plantea que "el educador debe cumplir con cuatro principios éticos que son: a) preparación intelectual y moral de alta calidad, b) respeto máximo por los derechos de los estudiantes, y una conducta personal intachable. Esto indica que para ejercer como educador, se requiere de unas condiciones específicas y que no basta con tener conocimientos o poseer un título académico, sino que va más allá de eso; se requiere de una conducta y formación puramente ética.

Es posible que esto lleve a reflexionar, sobre la realidad de los profesores universitarios. Lamentablemente, existen algunos docente a nivel mundial que no tienen en cuenta las normas éticas para ejercer su rol docente, ya que en muchos casos algunos estudiantes han vivenciado y a su vez testigos de los abusos de poder por parte de estos profesionales donde han sobornado y se dejan sobornar por los y las discentes con la finalidad de obtener la aprobación de una asignatura en particular, éste acto repudiable no sólo los afecta académicamente sino que tributa en la formación de un ser sin principios ni valores.

Por consiguiente, el docente universitario debe ser un profesional con un alto nivel de ética, para desempeñar una función efectiva y que sea cuestionada por las normas sociales e institucionales. En tal sentido, (Profesores et al., 1994) expresan que "Es preciso conjugar la libertad de cátedra con el carácter propio del centro universitario, ya que no todas las formas de ejercer la profesión, son compatibles con una visión moral confesional. Es de desear un diálogo interdisciplinar entre los profesores para que en la medida de lo posible y deseable, los alumnos no reciban en las asignaturas propias de sus carreras, criterios opuestos a los que se les transmiten en las asignaturas de formación". Esto indica la gran responsabilidad que descansa sobre los docentes del nivel superior, y si la universidad no da seguimiento y monitorea el trabajo de los docentes, puede darse el caso de que los profesores universitarios ejerzan una práctica totalmente fuera de lo establecido ética y profesionalmente.

Para que un docente actúe de manera ética debe estar formado en valores, para que de este modo actúe apegado fielmente a lo establecido por los códigos de ética institucional.

Según, lo que establece (Canalejas Pérez et al., 2005), "El profesor universitario ha de ser para sus estudiantes un modelo de actuación personal y profesional, un ejemplo que estimule a sus alumnos en el proceso de su construcción como persona, en el ejercicio de la profesión. Esto implica necesariamente el desarrollo del profesor como persona moral. Para ser modelo hay que ser coherente, tiene que mostrar correspondencia entre lo que dice y hace". Si se toma en consideración esta opinión, es de entender que la función del docente conlleva un alto grado de cumplimiento de la ética. 
Entendiéndose, que la ética en la práctica pedagógica del docente universitario debe estar fundamentada en la aplicación de teorías y métodos de enseñanza con el uso de recursos y medios que tengan la direccionalidad para llegar a sus estudiantes, deben llevar la seguridad para inducir un mejoramiento de su competencia profesional, que no se resuelve mediante el aprendizaje de meros saberes teóricos o puramente técnicos sino a la orientación esencial del quehacer educativo con el compromiso ético del docente.

Por lo tanto, un líder ético en el campo educativo tiene que conocer sus capacidades, perspectivas y sus limitaciones. Idealmente debe aprender a combinar las diferentes perspectivas en un estilo más integral y estratégico; aceptar sus propias limitaciones e incorporar a sus organizaciones aportes de fortalezas complementarias. Estos líderes conocen sus puntos fuertes, se esfuerzan por ampliarlos y estructuran equipos de trabajo que en conjunto ejercen el liderazgo, por lo menos, en las cuatro perspectivas: estructural, política, de recursos humanos y ética (Gómez Badillo, 2005).

En tal sentido, un líder ético en el componente educativo, debe ser capaz de ponerse al servicio de los demás; manteniendo a la vez la responsabilidad social, la objetividad, el conocimiento de las propias limitaciones. Incorporando idoneidad técnica, que se consigue con la formación académica y buscando generar junto a una visión completa de los problemas, una especialización en algunos de los campos en que se divide el servicio público y que lo proyectan hacia la construcción y fortalecimiento organizativo (Custo, 2004).

Partiendo de estas premisas es importante destacar, algunas cualidades que poseen los docentes con liderazgo ético:

- Compromisos del docente líder con su profesionalismo enmarcado en principios éticos y morales.

- Estimular la formación investigativa y el manejo tecnológico.

- Mostrar liderazgo en su área de formación.

- Explorar creativamente otros diseños pedagógicos, metodológicos y didácticos.

- Proponer otras formas de pensamiento.

- Crear un ambiente escolar adecuado y propicio para el liderazgo y la estimulación de otros talentos escondidos.

- Construir nuevos conocimientos a partir de los que se han alcanzado en la clase

- Fortalecer la estructura de la clase haciendo cultura de ella.

Por otra parte, (Martínez Martín et al., 2002) afirma que el docente se debe contemplar cómo. "Persona que acompaña al estudiante en el proceso de construcción de conocimiento, actitudes y valores en el que asume nuevas funciones que se expresan en su condición de gestar de información y guía del proceso de aprendizaje de sus estudiantes y que se resumen en su condición de modelo educativo".

La cita anterior, centra la educación superior como eje fundamental en la formación del futuro profesional, los cuales deben tener un perfil acondicionado a las exigencias y necesidades de la sociedad y para estar comprometido con el desarrollo social que hacen mención en el párrafo 
anterior. Para esto, es fundamental tener un formación integral (académica, tecnológica, científica, humanista entre otras competencias). De igual manera, tener bien fundamentado la ética profesional y ejecutarla en el accionar diario de la práctica pedagógica; con la intención de contribuir en la transformación del futuro profesional que está en formación.

Después, de indagar sobre esta temática a nivel internacional y en algunos países latino americano, el autor de este artículo contextualizó la investigación en la República Bolivariana de Venezuela, donde no escapa a esta realidad. A lo largo de la historia la educación en venezolana ha venido sufriendo cambios en el sistema educativo producto, de los cambios políticos, económicos, sociales y de la globalización. En la actualidad a pesar de los esfuerzos dados por el Estado Nacional de transformar la concepción de algunos docentes con visión capitalista, que sólo piensa en su propio bienestar y no les importa en incurrir en falta o delitos, porque adolece de principios éticos y volares.

- Contribuir con la interpretación teórica y la solución de los problemas de la sociedad y de la nación.

- Garantizar en todos los órdenes sociales el papel de agente de cambio y de transformación que bajo los principios y valores democráticos tiene la universidad venezolana para toda la colectividad nacional.

- Promover una conciencia de adscripción, identificación y servicio con la institución, la sociedad y la patria.

- Actuar como líder a objeto de defender, orientar y educar a los miembros de la sociedad venezolana, especialmente a los más desposeídos, en el proceso de instauración de la justicia social.

- Velar por el respeto de los principios contenidos en la declaración universal de los derechos humanos y de los de justicia y equidad contenidos en la constitución y demás leyes de la república.

- Fomentar los valores culturales, la identidad nacional, la libertad, la democracia, la responsabilidad y la solidaridad social.

\section{DESARROLLO}

¿Qué es lo que necesitan las universidades para crecer y no tener una muerte social? Quizás la respuesta más obvia sería contar con todos los recursos tecnológicos y económicos, sin embargo podemos ver que el crecimiento de una universidad y la permanencia en la sociedad se generan a través de las ofertas académicas que se reflejan al servicio a la comunidad.

Pero la tarea de un líder en la organización no es nada fácil, hacer que todo un grupo de personas se integre y trabaje en equipo conlleva a tener una buena comunicación, empatía, voluntad y compromiso con los colaboradores, lo que nos lleva a cuestionarnos ¿Qué es liderazgo? ¿Cuáles son los estilos del mismo? ¿Cómo es un buen líder? ¿Todo jefe es un líder? Existen tantas teorías, definiciones y perspectivas sobre este tema que de estudiarlo a fondo nos llevaría mucho tiempo. Pero empecemos desde el inicio, otra de las definiciones del liderazgo define el concepto como:

"El proceso mediante el cual un miembro del grupo (su líder) influye a los otros miembros hacia el logro de objetivos específicos grupales”. Entonces es claro poder ver qué líder no es toda 
persona que dirige una organización, sino aquel que puede influir en las personas para juntos lograr el objetivo común. ¿Qué cuál es la diferencia? Veamos, un jefe (digo jefe porque es el que está al mando de la organización) es aquella persona que solo ordena esperando que los miembros de la organización cumplan con sus deberes y objetivos, sin involucrarse directamente con ellos, mientras que un líder es aquella persona que motiva, escucha, atiende y comprende a los miembros de la organización sabiendo que sin el talento humano simplemente no se pude lograr los objetivos propuestos.

Una diferenciación muy acertada es la que hacen Roman y Ferrández en su libro Liderazgo y Coaching en la que nos dicen que:

1. El jefe maneja a sus coachees. El líder los capacita.

2. El jefe depende la autoridad. El líder, de la buena voluntad.

3. El jefe inspira temor. El líder, entusiasmo.

4. El jefe dice “уо”. El líder dice "nosotros".

5. El jefe arregla la culpa por el fracaso. El líder arregla el fracaso.

6. El jefe dice "vayan”. El líder dice "vamos”.

He aquí la diferencia entre lo que es ser un jefe y lo que es ser líder, por lo que lo es importante tener líderes capaces de sacar adelante la organización y no jefes que no hacen ningún cambio.

En cuanto a la intervención de las universidades por parte del CES, en cumplimiento a lo que demanda reglamento de creación, intervención y suspensión de universidades y escuela politécnica, el articulo 31 indica que "la intervención es una medida académica y administrativa de carácter cautelar y temporal, tendiente a resolver problemas que atenten contra el normal funcionamiento de la universidades y escuelas politécnica; mantener la continuidad de los procesos, asegurar y preservar la calidad de la gestión y, precautelar el patrimonio institucional...”. Bajo estas condiciones han sido intervenidas varias universidades en el Ecuador, en las cuales se ha detectado supuestas irregularidades como; transgredir el derecho a la gratuidad de la educación, corrupción, nepotismos, violencia y desvío de fondos.

De acuerdo al registro del CES, son 26 universidades de categoría E clasificadas por el CEAACES sobre la base del trabajo hecho por el CONEA desde 2009, las cuales ya no aparecen en noviembre del año 2015. De esta manera, de las 68 universidades de pre y posgrado en 2012, tenemos ahora 42, incluyendo a la ESPE, que está en proceso de evaluación, pues cambió a Universidad de las Fuerzas Armadas; 14 fueron cerradas de la categoría E. Se separa a las 10 que únicamente ofrecen pregrado en: 4 de categoría $\mathrm{B}, 4$ en la $\mathrm{C}$ y 2 en la $\mathrm{D}$. Y a las 3 que son de posgrado: 2 en la A y 1 en la B.

Esta reclasificación molesta a los que quedaron afuera o descendieron, pero en cada categoría hay novedades que levantaron críticas generalizadas. Las autoridades han insistido que solamente guardaron la categorización alfabética anterior como referencia, pues en realidad cada categoría responde a un nuevo proceso y eventualmente a una nueva métrica; sin embargo, parece obvio que la categoría A se redujo de 11 a 3; la B se incrementó de 9 a 18; la C subió de 13 a 14; y la D disminuyó de 9 a 6 . Claramente percibimos que 7 instituciones pasaron de la $\mathrm{A}$ a la $\mathrm{B}$ (esperando 
la evaluación de la Universidad de las Fuerzas Armadas). Y aquí es donde arde Troya, pues ninguna de las antiguas A reconoce su descenso. Pero hay novedades también en las otras categorías, considerando que en la D está la emblemática Universidad de Guayaquil, que junto a la Universidad Luis Vargas Torres de Esmeraldas se encuentran intervenidas.

Es importante evaluar a las universidades No obstante, hay algo en el ambiente universitario que nos hace titubear. Tal vez es un asunto no resuelto que se lo ha tratado con dobleces, ambigüedades, simulaciones y contradicciones. Pensamos que está bien que nos evalúen, pero no en la forma que lo han hecho. $\mathrm{Y}$ respaldamos dichas evaluaciones, siempre y cuando nos favorezcan y cuando se las realiza a otras instituciones. Y esto tiene más resistencia cuando hay personas con calidad profesional, pero externas a la academia, quienes son las encargadas del juicio y los escrutinios de la gestión universitaria.

Por el bien de la universidad, es necesario que la evaluación sea hecha por estos criterios de evaluación las cuales son: Academia, Eficiencia Académica, Investigación, Organización e Infraestructura, de tal manera de promover la eficacia de sus programas, que se refleja en el número y calidad de sus graduados y, en consecuencia, la eficiencia de sus procesos de enseñanza, para usar los menos recursos posibles.

Es necesario que el líder o los líderes de nuestra universidad cumplan con cada uno de estos parámetros para la acreditación, ya que de esta manera demostraran el grado de liderazgo en su dirección institucional

La Universidad Estatal del Sur de Manabí (UNESUM) se encuentra intervenida por el Consejo de Educación Superior (CES), tras denuncias de irregularidades cometidas en la institución. El rector de turno encargado, de nacionalidad cubana, realiza un trabajo colectivo para desarrollar proyectos integrales e internacionales, de esta manera aportar con la Re categorización de esta Universidad, resultados que se esperan, y se cumplan por el bienestar colectivo.

Con esta información en este artículo se pretende demostrar a la sociedad de Jipijapa y a toda la zona sur de Manabí y del resto que es necesario el cambio de líderes que direccionen a esta universidad, con el fin de que cumplan los objetivos y fines para la cual fue creada. Ante lo señalado, Las personas en su diario vivir se ven interpeladas por una serie de acciones y circunstancias; tanto en el ámbito personal como en el profesional, social e institucional; que requiere de una base ética y formación en valores sólida, que les permita enfrentar las diversas situaciones y dilemas éticos que se les presentan, de manera normal y equilibrada. En múltiples ocasiones la ética representa la diferencia entre alcanzar el éxito o llegar a un inminente fracaso. Del mismo modo la formación en valores conlleva, a un actuar apegado al cumplimiento de las normas sociales, profesionales e institucionales previamente establecidas, y cuando se procede en función a estas normas, la convivencia entre los seres humanos se hace más fácil, ya que prevalece el respeto, la armonía, cooperación, responsabilidad, amor y solidaridad, entre otros valores sociales y espirituales que ayudan a vivir en comunión con Dios y los demás seres del entorno natural y social en el que se interactúa .

En este documento se presenta la formulación del código ético personal del profesional y educador universitario; en el cual se incorpora la conceptualización ética, valores y las convicciones que han de guiar su conducción personal, familiar y profesional; a partir de:

a) la persona, 
b) su profesión,

c) familia,

d) participación comunitaria y social,

e) sentido de nación y de mundo,

f) concepción sobre ética ambiental, y económica.

A partir de este trabajo se espera un mayor afianzamiento de los valores positivos y comportamiento ético social, personal, profesional e institucional de los profesionales educativos. Se piensa en la ética en relación con las acciones individuales; pero existen razones de importancia por las cuales la calidad ética de las acciones institucionales tiene importancia en la vida ciudadana.

La postura ética de un individuo se ve afectada por la postura de sus grupos de referencia institucional. A su vez, los valores éticos institucionales influyen en modo considerable en su capacidad de desempeñarse de manera congruente. La raíz de la cuestión es, como ciertas responsabilidades individuales, derivan de la pertenencia de un individuo a cierta comunidad, y esas responsabilidades no existirían si tal individuo no perteneciera a ese grupo de interés.

Es necesario recordar como las organizaciones se componen de seres humanos, por lo que en ellas existe un procedimiento aceptado, a través del cual se toman decisiones, de suerte que el responsable de las decisiones tomadas, no es cada uno de los individuos de la organización, sino la organización en su conjunto (Cornelio et al., 2017).

A este respecto puede afirmarse como las cuestiones éticas en las organizaciones sociales, no pueden tratarse como si fueran problemas de optimización de decisiones; se trata de resolver el nivel en el cual se ubica el problema; una visión con los valores en juego, normas y mandatos sociales regidos por la cultura a considerar y agrega el deber ético; considerándolo como un imperativo, más no una obligación, sino una real y responsable decisión político-social, de cada ciudadano, que puede responder institucionalmente, pero también a su condición de ciudadano.

Por ello, las reformas de las instituciones de educación superior abren la vía en este sentido, para un poder de decisión pautado por las comunidades de interés; anteriormente sometidos a normas administrativas y de procedimientos de control definidos a priori. Surge de allí la inquietud del presente artículo, en cual tiene por objeto analizar el liderazgo ético, como soporte de gestión estratégica en universidades.

\section{RESULTADO}

De acuerdo a esto se ha logrado establecer que existe un desconocimiento sobre el manejo de la Administración en las universidades intervenidas producto de ello algunas con resultados nefastos y que hoy rinden cuentas en los estamentos judiciales del 'país. De la falta de liderazgo se ha hablado mucho, debido a su importancia en la historia de las organizaciones, ya sea social, política hasta incluso militares. Siempre ha sido un tema debatido entre grandes de los negocios, sin embargo, no ha importado si el líder en la organización nace o se hace, es indudable que gente líder es valorada en su empresa por ser impulsor y generador de valor agregado en ella. Algunos especialistas ven al liderazgo como una actividad amplia y visionaria que trata de discernir la 
competencia y valores característicos de una organización. Para determinar este propósito fue indispensable realizar la siguiente encuesta:

Encuesta dirigida a la comunidad universitaria docentes, estudiantes, trabajadores y ciudadanía. La encuesta fue elaborada por el Dr. Freddy Marcillo Merino, y la fuente de información la constituye la Población de la Universidad Estatal del Sur de Manabí.

1.- ¿¿Conoce usted el significado de liderazgo?
SI $62 \%$
NO 38\%

2.- ¿Cree usted que existan líderes en las universidades?

SI $20 \% \quad$ NO $80 \%$

3.- ¿¿Para usted, cuál de las siguientes cualidades debería tener un líder?

$\begin{array}{lc}\text { Ser impetuoso } & 0 \% \\ \text { Ser honesto } & 40 \% \\ \text { Ser soberbio } & 0 \% \\ \text { Ser responsable } & 60 \%\end{array}$

4.- ¿Cree usted que existe compromiso y responsabilidad por parte de los líderes en la Universidades Ecuatorianas?
Poco $5 \%$
Mucho 95\%
Nada $0 \%$

5.- Si tuviera que escoger un líder que características desearía que presentara escoja de la siguiente lista y la que no esté apúntela.

$\begin{array}{lc}\text { Claros } & 10 \% \\ \text { Disciplinado } & 05 \% \\ \text { Responsable } & 20 \% \\ \text { Constantes } & 10 \% \\ \text { Perseverantes } & 10 \% \\ \text { Honestos } & 25 \% \\ \text { Coherentes } & 10 \% \\ \text { Íntegros } & 10 \%\end{array}$

6.- ¿¿Conoce usted por qué hay Universidades intervenidas?
SI $20 \%$
NO $80 \%$

7.- ¿ ¿Cree usted que existan políticas de liderazgo en los predios Universitarios?

SI $28 \% \quad$ NO $72 \%$

8.- ¿Cómo desearía usted que fuera el carácter de un líder?

Proactivo (pensar antes de actuar) $100 \%$ Activo (actuar sin pensar) $0 \%$

9.- ¿Está usted de acuerdo en que el líder sea la persona que tome las decisiones sin pedir un consenso?
SI $48 \%$
NO $52 \%$

138 UNESUM-Ciencias. Publicación cuatrimestral. Vol. 4, No. 4 (Septiembre-Diciembre), Año 2020. 


\section{DISCUCIÓN}

El mundo se encuentra a la expectativa con el inicio de un nuevo siglo marcado por la globalización y una sociedad que, hoy por hoy, está experimentando transformaciones profundas en el contexto organizacional, espacios donde se está sustituyendo el concepto de producción industrial por el de los servicios, la información y el conocimiento. Los tiempos requieren apertura de mente, prontitud para acoger desafíos, audacia y seguridad para decidir en beneficio del bien común, sin evitar recordar con gratitud el pasado, vivir con pasión el presente y abrirse con confianza al futuro.

De las generalizaciones anteriores y en los límites del presente trabajo, se tiene como visión contribuir con la proyección de una toma de decisiones del docente desde una dimensión ético transformacional, como un compromiso personal, regional, local e institucional, en vías de generar conciencia para conservación y defensa del ser humano y de la sociedad objeto del presente estudio. Para tal efecto se plantea el siguiente problema: La humanidad ha progresado intelectualmente hasta extremos inimaginables, no obstante con preocupación se puede señalar que la sociedad está en crisis, porque la mayoría de las estructuras de las organizaciones que rigen el proceder de los ciudadanos se han deformado de tal manera que la complejidad y el caos han frustrado las buenas intenciones de quienes sin un mayor soporte político, económico o gubernamental; intentan adoptar decisiones desde una perspectiva que superponga lo ético, a lo puramente convencional. Por ello, se diseñó una investigación y una propuesta en el contexto de las organizaciones de educación superior, relacionadas con toma de decisiones ético transformacional del docente líder universitario.

El punto álgido busca vías de cambio y transformación desde otros escenarios: a) valores trascendentes desde la ética del ser como la fuerza capaz de orientar en función del diálogo y los acuerdos, las decisiones, a partir de su condición humana. b) El liderazgo docente centrado en la ética y en valores que reconozca la pluralidad y las diferencias en la manera de pensar, sentir, decir, decidir y actuar.

En este particular, es conveniente puntualizar algunas debilidades y amenazas en la toma de decisiones, detectadas mediante la entrevista individualizada realizada a docentes activos con 10 o más años y población con experiencia en la institución. Para ello, se han tomado algunos discursos textuales enmarcados en los supuestos iniciales del artículo, ante lo escrito es indispensable recomendar que, se debe motivar a los Docentes que sean parte de la Universidad para que se formen como líderes y que tomen la iniciativa, para mejorar la conducción de estamentos de Educación superior, y poder ser ejemplos de otras. De tomar conciencia sobre el compromiso a adquirir no podrán estar aptos para estos retos.

\section{CONCLUSIONES}

Se concluye que falta liderazgo en las entidades de educación superior, que la función es esencial, que depende fuertemente de la comunicación y de la motivación, utilizados de manera efectiva, permiten a un administrador obtener una participación activa y consciente en la consecución de los objetivos institucionales. La responsabilidad del administrador es crear a través de la comunicación, de la motivación y del liderazgo, un ambiente propicio tal que otros lo encuentren 
satisfactorio y atractivo para desarrollarse. El liderazgo, la comunicación y la motivación son también esenciales para ayudar a que los demás acepten los cambios necesarios en la organización y, así mismo, para crear una atmósfera de trabajo estable y tranquilo, tener dirección visionaria, pasión, integridad, conocerse a sí mismo, candor, madurez e integridad, que son cualidades y Muchos otras características que tienen los líderes: coraje (fuerza interior), moral (valor para aceptar la responsabilidad), físico (valor para enfrentar los retos), potencia del intelecto (juicio razonado para la toma de decisiones), captación a primera vista (capacidad de reconocer una situación rápidamente), determinación (capacidad de mantener una decisión una vez adoptada), características que necesitan los directivos para administrar con . Eficacia eficiencia y efectividad.

\section{REFERENCIAS BIBLIOGRÁFICAS}

Angulo, N., \& Acuña, I. (2005). Ética del docente. Revista Educación en valores, 2, 1.

Canalejas Pérez, M., Martínez Martín, M., Pineda Ginés, M., Vera Cortés, M. L., Soto González, M., Martín Marino, Á., \& Cid Galán, M. (2005). Estilos de aprendizaje en los estudiantes de enfermería. Educación médica, 8(2), 33-40. http://scielo.isciii.es/pdf/edu/v8n2/original2.pdf

Cornelio, O. M., Díaz, P. M. P., \& Fonseca, B. B. (2017). Estrategia metodológica para disminuir el impacto medioambiental de la tecnología obsolescente. REFCalE: Revista Electrónica Formación y Calidad Educativa. ISSN 1390-9010, 5(2), 99-118.

Cornelio, O. M., \& Gulín, J. G. (2018). Modelo para la evaluación de habilidades profesionales en un Sistema de Laboratorios a Distancia. Revista Científica, 3(33), 1.

Custo, T. (2004). UBICACIÓN EN EL PLAN DE ESTUDIO Universidad Nacional de Córdoba]. https://sociales.unc.edu.ar/sites/default/files/TEORIA,\%20ESPACIOS\%20Y\%20ESTRATEGIAS \%20DE\%20INTERVENCION\%20I\%20\%20B\%20(GRUPAL).pdf

Gómez Badillo, J. (2005). Liderazgo: rasgos, funciones y estilos Universidad de La Sabana]. https://intellectum.unisabana.edu.co/bitstream/handle/10818/4621/130817.pdf?sequence=1\&isAll owed $=\mathrm{y}$

Guzmán, W. (2004). Las carreras humanísticas en Venezuela Tesis de grado, Mención Publicación. Caracas: Escuela de Educación ....].

Marques, L. P., Lauro, B. R., da Silva, C. F., de Oliveira, M. L. D. Z., Klaegen, N. A., \& da Silva Monteiro, S. (2005). Educação Infantil como prática de transformação social. Interagir: pensando extensão(7), 97. https://www.epublicacoes.uerj.br/index.php/interagir/article/download/21389/15507

Martínez Martín, M., Buxarrais Estrada, M. R., \& Esteban Bara, F. (2002). La universidad como espacio de aprendizaje ético. Revista Iberoamericana de Educación (OEI), 2002, num. 29, p. 17-43. http://diposit.ub.edu/dspace/bitstream/2445/56963/1/565428.pdf

Profesores, U. P. C. S. d., Fernández, J. L. F., \& Hortal, A. (1994). Ética de las profesiones. UPCO. 